\title{
Exploring MR regression patterns in rectal cancer during neoadjuvant radiochemotherapy with daily $\mathrm{T} 2$ - and diffusion-weighted MRI
}

T. Bostel ${ }^{1,2^{*}{ }^{\prime}}$, C. Dreher ${ }^{3,4 \dagger}$, D. Wollschläger ${ }^{5}$, A. Mayer ${ }^{2}$, F. König $^{3}$, S. Bickelhaupt ${ }^{6,7}$, H. P. Schlemmer ${ }^{3}$, P. E. Huber ${ }^{1,8}$, F. Sterzing ${ }^{1,8,9}$, P. Bäumer ${ }^{3,10}$, J. Debus ${ }^{1,8}$ and N. H. Nicolay ${ }^{1,11^{*}}$

\begin{abstract}
Background: To date, only limited magnetic resonance imaging (MRI) data are available concerning tumor regression during neoadjuvant radiochemotherapy $(\mathrm{RCT})$ of rectal cancer patients, which is a prerequisite for adaptive radiotherapy (RT) concepts. This exploratory study prospectively evaluated daily fractional MRI during neoadjuvant treatment to analyze the predictive value of MR biomarkers for treatment response.

Methods: Locally advanced rectal cancer patients were examined with daily MRI during neoadjuvant RCT. Contouring of the tumor volume was performed for each MRI scan by using T2- and diffusion-weighted-imaging (DWI)-sequences. The daily apparent-diffusion coefficient (ADC) was calculated. Volumetric and functional tumor changes during RCT were analyzed and correlated with the pathological response after surgical resection.

Results: In total, $171 \mathrm{MRI}$ scans of eight patients were analyzed regarding anatomical and functional dynamics during RCT. Pathological complete response ( $P C R$ ) could be achieved in four patients, and four patients had a pathological partial response (pPR) following neoadjuvant treatment. T2- and DWI-based volumetry proved to be statistically significant in terms of therapeutic response, and volumetric thresholds at week two and week four during RCT were defined for the prediction of $\mathrm{PCR}$. In contrast, the average tumor ADC values widely overlapped between both response groups during RCT and appeared inadequate to predict treatment response in our patient cohort.

Conclusion: This prospective exploratory study supports the hypothesis that MRI may be able to predict pCR of rectal cancers early during neoadjuvant RCT. Our data therefore provide a useful template to tailor future MRguided adaptive treatment concepts.
\end{abstract}

Keywords: MRI, Rectal cancer, Chemoradiotherapy, Oncologic imaging, Pathological response, IGRT

\footnotetext{
*Correspondence: tilman.bostel@unimedizin-mainz.de; nils.nicolay@uniklinik-

freiburg.de

Bostel T and Dreher $C$ are Shared first authors

${ }^{\dagger}$ T. Bostel and C. Dreher contributed equally to this work.

'Clinical Cooperation Unit Radiation Oncology, German Cancer Research Center (DKFZ), Im Neuenheimer Feld 280, 69120 Heidelberg, Germany

Full list of author information is available at the end of the article
}

(c) The Author(s). 2020 Open Access This article is licensed under a Creative Commons Attribution 4.0 International License, which permits use, sharing, adaptation, distribution and reproduction in any medium or format, as long as you give appropriate credit to the original author(s) and the source, provide a link to the Creative Commons licence, and indicate if changes were made. The images or other third party material in this article are included in the article's Creative Commons licence, unless indicated otherwise in a credit line to the material. If material is not included in the article's Creative Commons licence and your intended use is not permitted by statutory regulation or exceeds the permitted use, you will need to obtain permission directly from the copyright holder. To view a copy of this licence, visit http://creativecommons.org/licenses/by/4.0/ The Creative Commons Public Domain Dedication waiver (http://creativecommons.org/publicdomain/zero/1.0/) applies to the data made available in this article, unless otherwise stated in a credit line to the data. 


\section{Background}

Colorectal cancer constitutes the third most common malignant tumor disease with an estimated global incidence of more than one million people per year [1]. The highest annual incidence rates of colorectal cancer are recorded in the developed countries affecting more than 40 per 100.000 people [1]. In the last decades, the cancer-specific mortality rates of rectal cancer have declined significantly owing to considerable advances in treatment as well as improved diagnostic techniques and extended screening measures $[2,3]$.

Radical resection of the rectum remains the mainstay of curative treatment for rectal cancer; but for locally advanced disease, multimodal therapeutic approaches including radiotherapy (RT) have resulted in significantly improved local control, but no overall survival benefit compared to surgery alone $[4,5]$. Neoadjuvant radiochemotherapy (RCT) followed by surgery has emerged as a standard of care for patients with locally advanced rectal cancers, leading to pathologically complete response (pCR) rates of $11-31 \%$ [6-8]. Complete responders after neoadjuvant treatment have a favourable outcome with a 5-year disease-free survival of about $83 \%$, making tailored and potentially organ-preserving treatments relevant for these patients in order to reduce therapy-related morbidity and hence improve long-term quality of life [9-11]. In turn, non-responders need to be identified as early as possible, as these patients may benefit from modified and potentially more aggressive treatment concepts and may not be suitable candidates for organ preservation strategies [12].

Consequently, an early prediction of tumor response to neoadjuvant treatment is of special interest. Its assessment may require the monitoring of predictive molecular biomarkers as well as advanced imaging during the course of $\mathrm{RCT}$, although suitable strategies are lacking to date. In recent years, magnetic resonance imaging (MRI) has emerged as the most promising imaging procedure for the prediction of treatment response during and after neoadjuvant RCT [13, 14]. However, available studies are difficult to compare due to inconsistent imaging time points and assessment of different MRI parameters [15-18].

To the best of our knowledge, no data have been published concerning daily anatomical and functional changes of MRI during RCT in rectal cancer. In recent years, our group demonstrated the safety and feasibility of a shuttlebased off-line approach for realizing daily MR-guided radiotherapy $[19,20]$. This analysis aimed to measure daily evolutions in tumor volume and apparent diffusion coefficient (ADC), and correlate these findings to the final pathological response outcome to gain new insights into detailed MR regression patterns during neoadjuvant RCT.

\section{Material and methods}

\section{Patients}

Our manuscript analyzed data from a prospective trial investigating daily MRI in treatment position as a means of off-line MR-guided radiotherapy [19, 20]. In this study, 8 patients with locally advanced rectal adenocarcinoma were enrolled between October 2013 and June 2017. All patients completed neoadjuvant RCT. Pathological complete response (pCR; Dworak regression grade 4) could be achieved in 4 patients, while the rest of the patients had a pathological partial response (pPR; Dworak regression grade 1-3) to neoadjuvant treatment [21]. Detailed patient characteristics are provided in Table 1. The trial was carried out in accordance with the Declaration of Helsinki (7th revision) and was approved by the independent ethics commission of the Medical Faculty of the University of Heidelberg (S-144/2013). All patients provided written informed consent prior to inclusion in this trial.

\section{Patient immobilization and treatment planning}

All patients received daily short MR scans in treatment position prior to irradiation with the same immobilization equipment used for RT to image the morphological and functional changes of the tumorous tissue during the treatment. The transfer of patients between the MR device and the linear accelerator was realized with a shuttle system (Zephyr system, Diacor, Salt Lake City, USA) [19].

Treatment planning was performed using the RayStation $^{\text {TM }}$ treatment planning system (RaySearch, Stockholm, Sweden). Gross tumor volume (GTV) was defined as the morphological tumor volume, visible on post-contrast computed tomography and co-registered MRI scans. The clinical target volume (CTV) was defined as proposed by Valentini et al. [22]. The planning target volume (PTV) was defined as the CTV with addition of 5 $\mathrm{mm}$ in the horizontal and the cranio-caudal plane.

All patients received 50.4 Gy in 28 fractions and concurrent chemotherapy with 5 -fluoruracil $\left(300 \mathrm{mg} / \mathrm{m}^{2}\right.$ body surface area daily, administered intravenously via a port catheter system).

Inverse treatment planning was used for intensitymodulated RT. Treatment was carried out using a $6 \mathrm{MV}$ linear accelerator (Siemens Artiste, Erlangen, Germany). Prior to each treatment fraction, position verification imaging was performed using KV cone-beam CT scans.

\section{MR imaging}

Daily MRI examinations were performed for all patients in treatment position immediately before treatment using a $1.5 \mathrm{~T}$ MRI scanner (Magnetom Symphony, Siemens Healthcare, Erlangen, Germany) [14]. The MRI protocol included a high-resolution coronal T2-space sequence $(\mathrm{TR}=2 \mathrm{~s} ; \mathrm{TE}=125 \mathrm{~ms} ;$ voxel size $=1.2 \times 1.0 \times$ $1.12 \mathrm{~mm}^{3}$; acquisition matrix $\left.448 \times 288 \times 314\right)$, a transverse T1-vibe sequence $(\mathrm{TR}=10 \mathrm{~ms}, \mathrm{TE}=5 \mathrm{~ms}$, voxel size $=$ $2.0 \times 2.0 \times 2.0 \mathrm{~mm}^{3}$; acquisition matrix $=228 \times 287 \times 378$ ) and transverse DWI. For tumor contouring, transverse and sagittal images were reconstructed from the coronal 
Table 1 Patients' characteristics

\begin{tabular}{|c|c|c|}
\hline Characteristics & Value & Percent \\
\hline \multicolumn{3}{|l|}{ Age (y) } \\
\hline - Median & 63.5 & \\
\hline - Range & $51-72$ & \\
\hline \multicolumn{3}{|l|}{ Gender } \\
\hline -Female & 5 & 62.5 \\
\hline -Male & 3 & 37.5 \\
\hline \multicolumn{3}{|l|}{ Clinical stage before treatment (n) } \\
\hline$-\mathrm{cT} 2 \mathrm{~N} 1$ & 1 & 12.5 \\
\hline -cT3 N0-2 & 7 & 87.5 \\
\hline \multicolumn{3}{|l|}{ Pathological stage (n) } \\
\hline -ypT0 NO (pCR) & 4 & 50.0 \\
\hline -урT2 No-1 & 2 & 25.0 \\
\hline -урT3 No-2 & 2 & 25.0 \\
\hline \multicolumn{3}{|l|}{ Dworak tumor regression grade (TRG) } \\
\hline -Dominant tumor mass with obvious fibrosis (TRG $1=$ minimal regression) & 2 & 25.0 \\
\hline -Dominantly fibrotic changes with few tumor cells or groups (TRG $2=$ moderate regression) & 1 & 12.5 \\
\hline -Very few tumor cells (TRG $3=$ near pCR) & 1 & 12.5 \\
\hline -No tumor cells (TRG $4=$ pCR) & 4 & 50.0 \\
\hline \multicolumn{3}{|l|}{ Initial tumor volume (ml) } \\
\hline -Median & 33.5 & \\
\hline -Range & $14-95$ & \\
\hline \multicolumn{3}{|l|}{ Total treatment time $(d)$} \\
\hline -Median & 40 & \\
\hline -Range & $37-43$ & \\
\hline
\end{tabular}

Abbreviations: $y$ years, $n$ number, $p C R$ pathological complete response, TRG tumor regression grade, $\mathrm{ml}$ milliliters, $d$ days

T2-space sequence. DWI was performed in free breathing and in case of baseline MRI prior to administration of contrast agent. The following parameters were used for DWI acquisition: TR $=7.54 \mathrm{~s} ; \mathrm{TE}=96 \mathrm{~ms}$; FOV read/ phase $280 \mathrm{~mm} / 76.5 \%$; acquisition matrix 102/0/0/78; Voxel size $2.7 \times 2.7 \times 3.2 \mathrm{~mm}$; slice thickness $3.2 \mathrm{~mm}$; bandwidth $1442 \mathrm{~Hz} / \mathrm{Px}$; b-values 0 and $1000 \mathrm{~s} / \mathrm{mm}^{2}$, gradient mode 3 -scan trace; 50 slices in 1 step; 6 min. ADC maps were calculated by a monoexponential fitting model. For a total of 171 of 224 treatment fractions (76\%), MRI scans could be conducted and were analyzed regarding anatomical and functional dynamics during RCT.

\section{Data analysis}

GTV was measured using T2-weighted and diffusionweighted images (DWI). The DWI ROIs were semiautomatically segmented at the setting of $b=1000 \mathrm{~s} / \mathrm{mm}^{2}$ and by the use of the "Medical Imaging Interaction Toolkit" (MITK) transferred to co-registered images at the setting of $\mathrm{b}=0 \mathrm{~s} / \mathrm{mm}^{2}$ [23]. Co-registered $\mathrm{T} 1$ and $\mathrm{T} 2$ sequences were used to assist visually locating the morphological lesion. To ensure that only areas with restricted diffusivity were considered for volumetry, a threshold tool of the MITK software was used. Further segmentation was performed on each slice, in all sections and within inner limits to reduce partial volume effects. After semi-automatic generation of the DWI-based tumor volumes, these were checked by the two radiologists and manually corrected if necessary. ADC values in the DWI ROIs were calculated by a monoexponential fitting model, based on a software code developed in MATLAB (MathWorks, Natick, Massachusetts).

Volumetric and functional MR data were defined by the consensus of two diagnostic radiologists. Both radiologists were blinded for the pathological response.

\section{Statistical analysis}

Tumor volume at each radiation fraction of RCT was compared with the corresponding volume at the first and previous treatment fraction. Furthermore, tumor volumes and ADC values at baseline as well as at weeks 2 and 4 after initiation of RT were compared between the response groups (i.e., pathological complete or partial response) using the Wilcoxon-Mann-Whitney test with a significance level of $p<0.05$. These time points were 

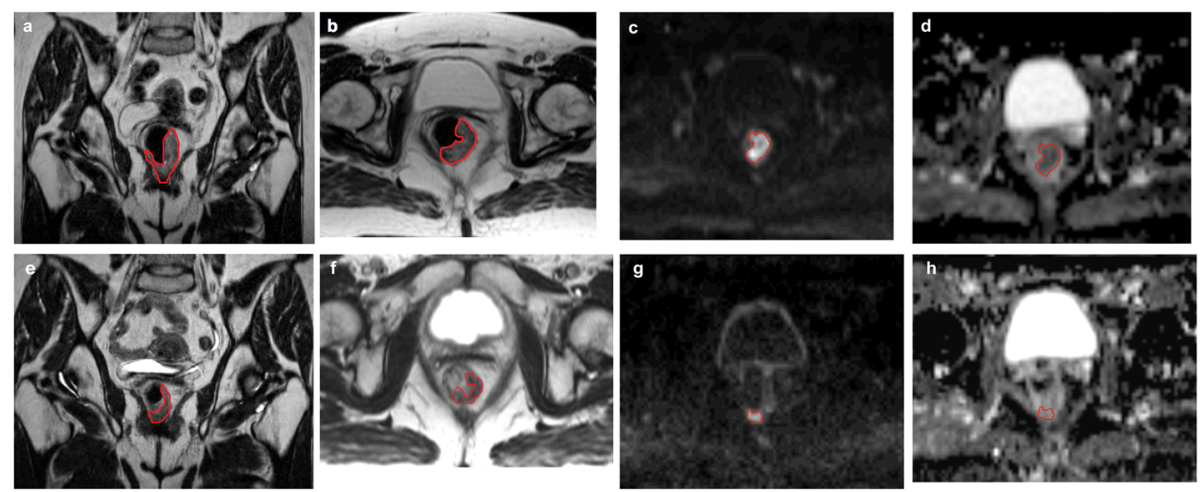

Fig. 1 Example of tumor regression in a single patient between the first and last fraction of neoadjuvant treatment. Distinct decrease in gross tumor volume (GTV; red outlined) is evident ( $\mathbf{a}+\mathbf{b}$ : coronal and transverse T2-weighted images at baseline, $\mathbf{e}+\mathbf{f}$ : coronal and transverse T2weighted images at the end of neoadjuvant RCT). The diffusion-restricted area within the GTV (red) also declines until end of RCT, indicating the treatment-related cell depletion ( $\mathbf{c}$ d: transverse DWI sequence obtained with a b-value of $1000 \mathrm{~s} / \mathrm{mm}^{2}$ and corresponding ADC-map at baseline, $\mathbf{g}+\mathbf{h}$ : transverse DWI sequence ( $\mathrm{b}$-value $=1000$ ) and ADC-map at the end of neoadjuvant RCT; the segmented GTV (red) on the ADCmaps is given to highlight the tumor, however segmentation for the ADC calculation has been performed on high b-value images

chosen because they offered complete data. Due to the exploratory nature of the study, no formal adjustment for multiple testing was carried out. Statistical analysis was performed by the $\mathrm{R}$ software package, version 3.5.1 ( $\mathrm{R}$ Core Team 2018, Vienna, Austria).

\section{Results}

Volumetric analysis T2-based volumetry

All patients exhibited a distinct volume reduction of the tumor during the course of RCT (see Figs. 1 and 2) with most pronounced changes in patients reaching a pCR (see Table 2): From baseline to the end of neoadjuvant treatment, the average T2-based tumor volume decreased from $39 \mathrm{~cm}^{3}$ (range $14-95 \mathrm{~cm}^{3}$ ) to $10.9 \mathrm{~cm}^{3}$ (range $0-28 \mathrm{~cm}^{3}$ ) Patients with a pCR had a significant lower absolute T2-tumor volume at the beginning of RCT as well as after 2 and 4 weeks of treatment (fraction 11 and fraction 22) than those patients with a pPR (see Fig. 2 and Table 2). The T2-based tumor volumes of patients with pCR and pPR averaged $19.3 \mathrm{~cm}^{3}$ (range $14-30 \mathrm{~cm}^{3}$ ) and $58.2 \mathrm{~cm}^{3}$ (range $37-95 \mathrm{~cm}^{3}$ ) prior to

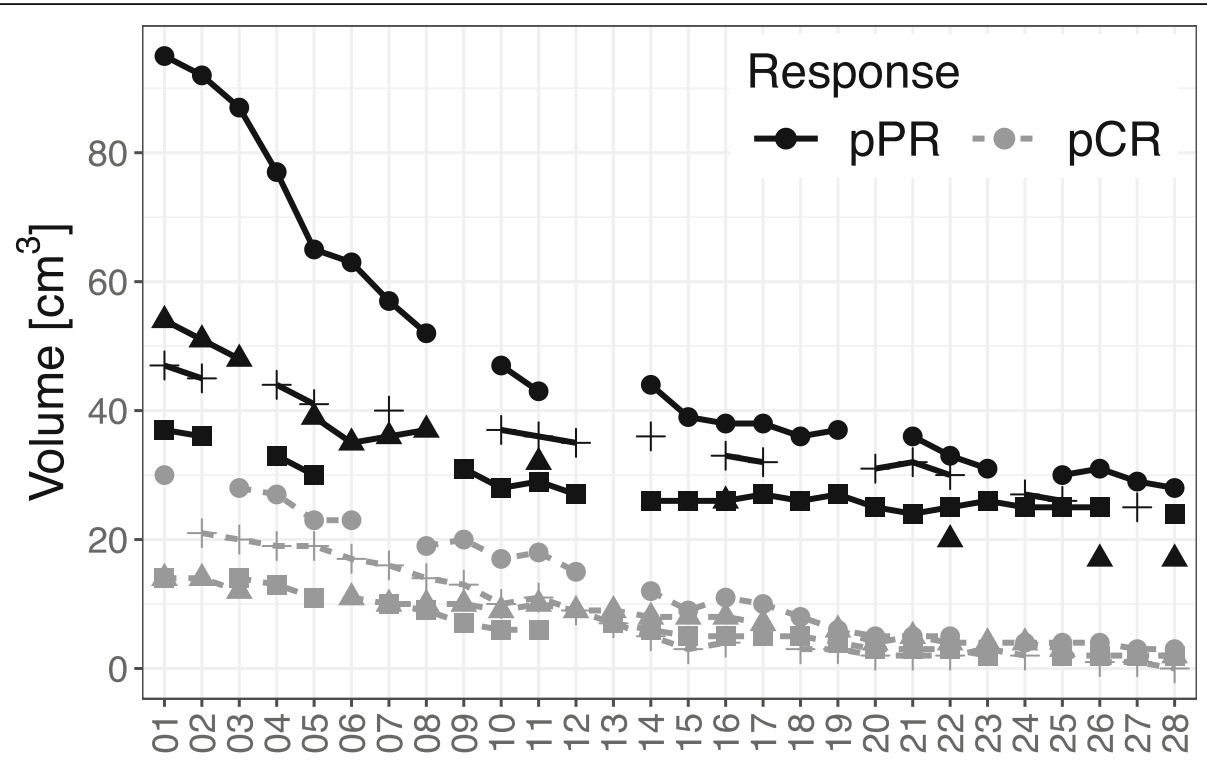

Fraction

Fig. 2 Separate presentation of gross tumor volume dynamics for each patient during neoadjuvant RCT (based on T2w sequence). Between the complete and partial pathological responders ( $\mathrm{PCR}$ and $\mathrm{pPR}$ ) distinct differences of absolute tumor volumes were evident at baseline as well as over the whole course of RCT 
Table 2 Comparison of T2-based tumor volumetry between response groups

\begin{tabular}{|c|c|c|c|c|c|c|c|c|c|c|c|c|}
\hline & \multicolumn{12}{|c|}{ T2-based tumor volumetry (ml) } \\
\hline & \multicolumn{6}{|l|}{$\overline{\mathrm{pCR}}$} & \multicolumn{6}{|l|}{ pPR } \\
\hline & Mean & Min & Max & Mean rel. to BL (\%) & Mean rel. to PM (ml) & $n$ & Mean & Min & Max & Mean rel. to BL (\%) & Mean rel. to PM (\%) & \\
\hline Fx 1 & 19.3 & 14 & 30 & 100 & & 3 & 58.2 & 37 & 95 & 100 & & \\
\hline Fx 2 & 17.5 & 14 & 21 & 100 & 0 & 2 & 56.0 & 36 & 92 & 96.1 & 4 & \\
\hline Fx 3 & 18.5 & 12 & 28 & 93.0 & 6.5 & 4 & 67.5 & 48 & 87 & 90.2 & 5.5 & \\
\hline Fx 4 & 19.7 & 13 & 27 & 91.4 & 5.3 & 3 & 51.3 & 33 & 77 & 88.0 & 7 & \\
\hline Fx 5 & 17.7 & 11 & 23 & 77.6 & 10 & 3 & 43.8 & 30 & 65 & 77.2 & 12.8 & \\
\hline Fx 6 & 17.0 & 11 & 23 & 77.6 & 6.3 & 3 & 49.0 & 35 & 63 & 65.6 & 6.5 & \\
\hline Fx 7 & 12.0 & 10 & 16 & 71.4 & 8 & 3 & 44.3 & 36 & 57 & 70.6 & 4 & \\
\hline Fx 8 & 13.0 & 9 & 19 & 66.3 & 9.8 & 4 & 44.5 & 37 & 52 & 61.6 & 4.5 & \\
\hline Fx 9 & 12.5 & 7 & 20 & 62.7 & 7.3 & 4 & 31.0 & 31 & 31 & 83.8 & 0 & \\
\hline Fx 10 & 10.5 & 6 & 17 & 54.6 & 15.5 & 4 & 37.3 & 28 & 47 & 68.0 & 9 & \\
\hline Fx 11 & 11.3 & 6 & 18 & 58.1 & 0 & 4 & 35.0 & 29 & 43 & 64.9 & 6.5 & \\
\hline Fx 12 & 11.0 & 9 & 15 & 57.1 & 15 & 3 & 31.0 & 27 & 35 & 73.7 & 5 & \\
\hline Fx 13 & 7.7 & 7 & 9 & 57.1 & 7.3 & 3 & NA & - & - & NA & NA & \\
\hline Fx 14 & 7.8 & 5 & 12 & 46.7 & 18.5 & 4 & 35.3 & 26 & 44 & 64.4 & 1.3 & \\
\hline Fx 15 & 6.3 & 3 & 9 & 41.0 & 20.5 & 4 & 32.5 & 26 & 39 & 55.7 & 5.5 & \\
\hline Fx 16 & 7.0 & 4 & 11 & 43.2 & 0 & 4 & 30.8 & 26 & 38 & 57.2 & 7.5 & \\
\hline Fx 17 & 7.3 & 5 & 10 & 39.7 & 7 & 3 & 32.3 & 27 & 38 & 60.4 & 1 & \\
\hline Fx 18 & 5.3 & 3 & 8 & 31.2 & 15 & 3 & 31.0 & 26 & 36 & 54.1 & 4.5 & \\
\hline Fx 19 & 4.8 & 3 & 6 & 30.5 & 14.8 & 4 & 32.0 & 27 & 37 & 56.0 & 0 & \\
\hline Fx 20 & 3.5 & 2 & 5 & 22.2 & 27 & 4 & 28.0 & 25 & 31 & 66.8 & 5 & 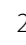 \\
\hline Fx 21 & 3.8 & 2 & 5 & 24.6 & 0 & 4 & 30.7 & 24 & 36 & 57.0 & 2.3 & \\
\hline Fx 22 & 3.5 & 2 & 5 & 22.2 & 5 & 4 & 27.0 & 20 & 33 & 50.8 & 9.3 & \\
\hline Fx 23 & 3.0 & 2 & 4 & 21.4 & 11 & 3 & 28.5 & 26 & 31 & 51.5 & 3 & 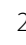 \\
\hline Fx 24 & 3.3 & 2 & 4 & 20.9 & 17.8 & 3 & 26.0 & 25 & 27 & 62.5 & 7 & 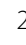 \\
\hline Fx 25 & 3.0 & 2 & 4 & 16.4 & 8.3 & 3 & 27.0 & 25 & 30 & 51.5 & 2.3 & \\
\hline Fx 26 & 2.3 & 1 & 4 & 13.8 & 16.7 & 3 & 24.3 & 17 & 31 & 43.9 & 5 & . \\
\hline Fx 27 & 2.0 & 1 & 3 & 12.9 & 14.5 & 4 & 27.0 & 25 & 29 & 41.9 & 5 & . \\
\hline Fx 28 & 1.8 & 0 & 3 & 12.9 & 25 & 4 & 23.0 & 17 & 28 & 41.9 & 2.3 & 3 \\
\hline
\end{tabular}

Abbreviations: $\mathrm{m} /$ milliliters, $p C R$ pathological complete response, $p P R$ pathological partial response, rel. relative, $B L$ baseline, $P M$ previous measurement, $M i n$ minimum, Max maximum, $F x$ fraction, NA not analyzable, i.e. no values existent

$\operatorname{RCT}\left(p=0.03\right.$ ), $11.3 \mathrm{~cm}^{3}$ (range $6-18 \mathrm{~cm}^{3}$ ) and $35.0 \mathrm{~cm}^{3}$ (range $29-43 \mathrm{~cm}^{3}$ ) at 2 weeks after the initiation of RCT $\left(p<0.01\right.$ ), and $3.5 \mathrm{~cm}^{3}$ (range $2-5 \mathrm{~cm}^{3}$ ) and 27.0 $\mathrm{cm}^{3}$ (range $\left.20-33 \mathrm{~cm}^{3}\right)$ at week 4 during RCT $(p<$ $0.01)$, respectively. Until the end of neoadjuvant treatment, the mean relative tumor shrinkage rate was $76.7 \%$ in the overall study population, $87.1 \%$ in the pCR group and $58.1 \%$ in the pPR group as compared to the baseline tumor volume. The fastest tumor shrinkage rate relative to the baseline volume was observed during the first 2 weeks of treatment independently from the pathological treatment response. However, in the following weeks distinct differences of tumor shrinkage were evident between both response groups (see Figs. 1 and 3).
In the overall study population and the subgroups of patients with $\mathrm{pCR}$ and $\mathrm{pPR}$, average tumor volume decreased by $2.7,3.1$ and $2.1 \%$, respectively, per radiation fraction compared to baseline volume (please see Fig. 4 and Table 2 for detailed description of daily rectal tumor volume changes). In the overall study population and the subgroups of patients achieving pCR and pPR, tumor volume declined on average by $7.0,9.0$ and $4.2 \%$ from one fraction to the next. The biggest differences of daily tumor shrinkage between both response groups were observed in the last 3 weeks of $\mathrm{RCT}$; in the pCR group, average daily tumor shrinkage was steadily increasing during RCT, while in the pPR group the tumor shrinkage rates were 


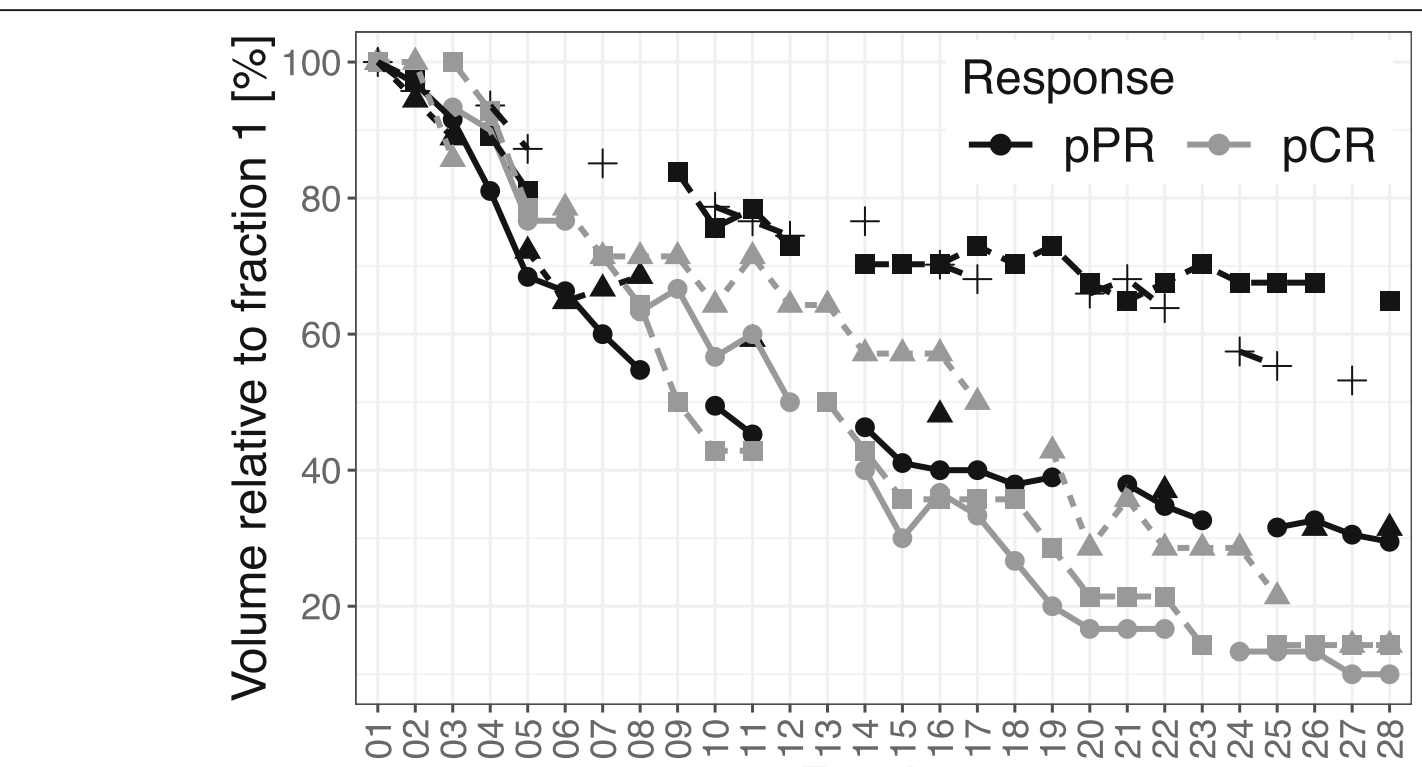

Fraction

Fig. 3 Separate presentation of gross tumor volume changes during RCT relative to the first fraction of treatment for each patient (based on T2W sequence). While in the first 2 weeks all patients exhibited substantial tumor shrinkage, distinct differences were evident between pathological complete and partial responders ( $\mathrm{PCR}$ and $\mathrm{pPR}$ ) in the further course of RCT

considerably lower and decreasing until the end of treatment (see Fig. 5 and Table 2).

\section{DWI-based volumetry}

In general, the tumor volumes assessed using DWI $(b=$ $1000 \mathrm{~s} / \mathrm{mm}^{2}$ ) were lower than those assessed by T2-based imaging (see Table 3). At baseline, no significant difference in average DWI-based tumor volumes between the pCR and pPR groups was evident $\left(7.8\right.$ vs. $23.4 \mathrm{~cm}^{3}, p=$ 0.10). In accordance with the T2-based volume assessment, there were distinct volume changes during RCT (see Table 3). From baseline to the end of neoadjuvant

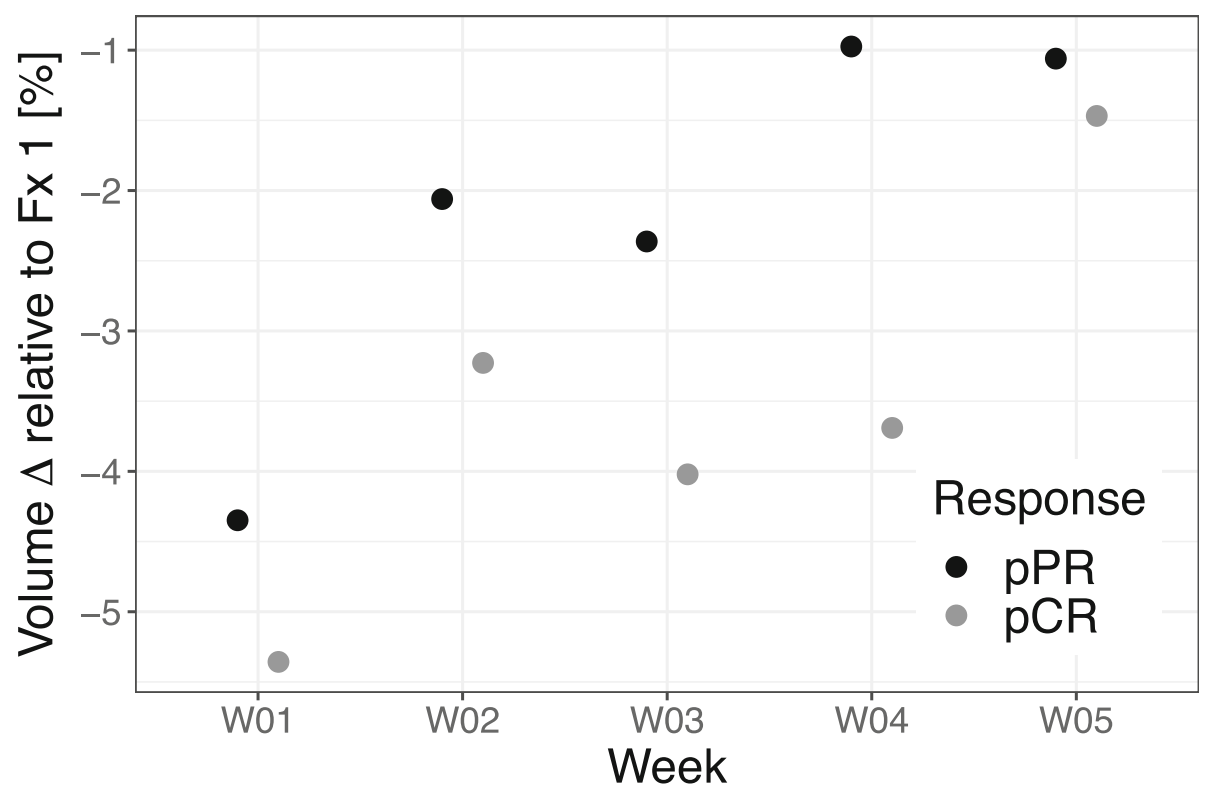

Fig. 4 The points in the figure represent average daily volume changes per week relative to the first fraction of neoadjuvant RCT (baseline) for complete and partial pathological responders (pCR and pPR) separately. Complete and partial pathological responders (pCR and pPR) differed substantially, particularly in the first 4 weeks of treatment 


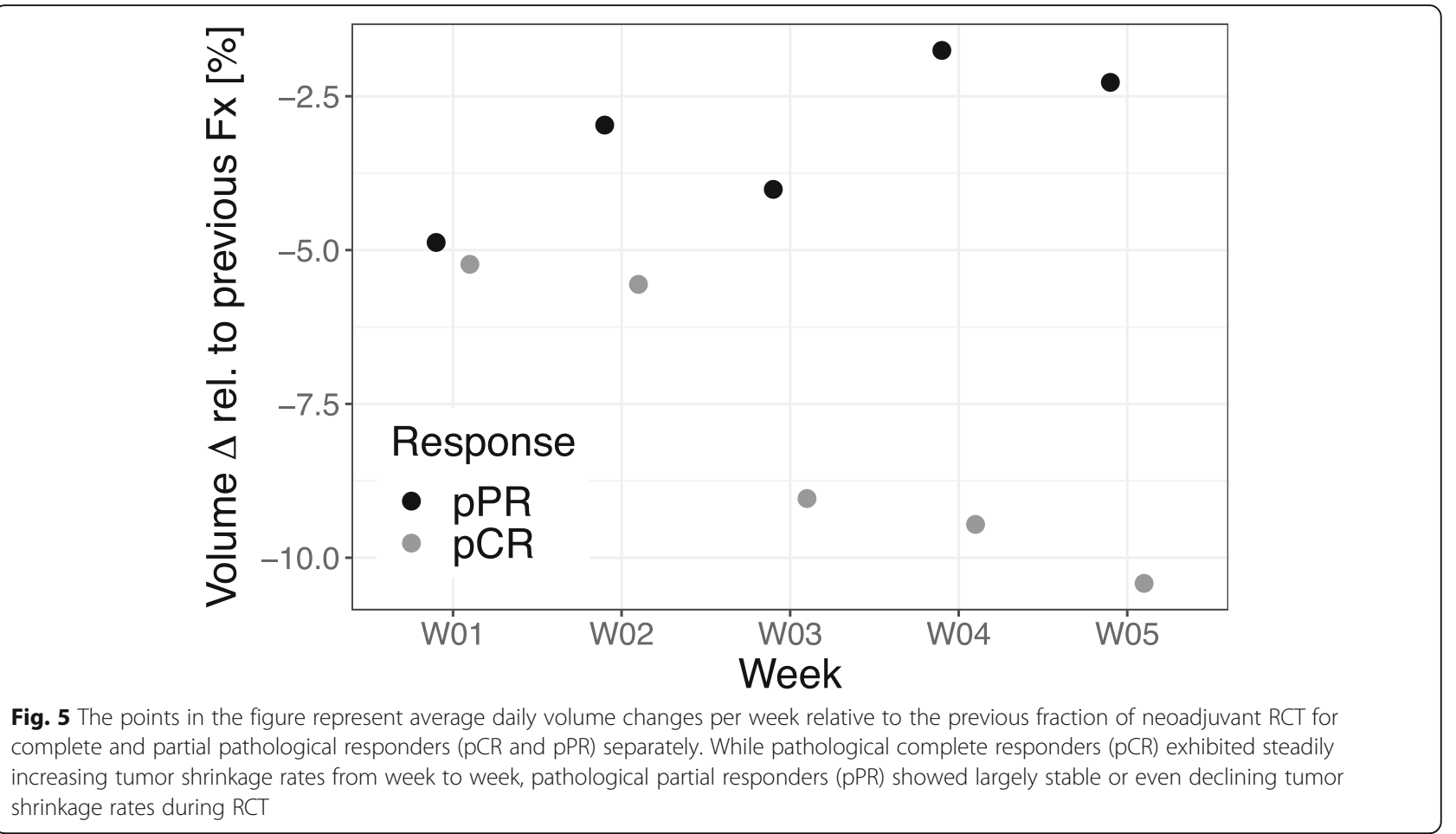

treatment, the average DWI-based tumor volume of the overall study population decreased from $16.7 \mathrm{~cm}^{3}$ (range $5.4-38.3 \mathrm{~cm}^{3}$ ) to $5.0 \mathrm{~cm}^{3}$ (range $0-28 \mathrm{~cm}^{3}$ ) After 2 weeks of neoadjuvant treatment, DWI-based tumor volumes were lower in the pCR group compared to the pPR group (pCR vs. pPR: average volume $2.0 \mathrm{~cm}^{3}$ vs. 11.9 $\left.\mathrm{cm}^{3}, p=0.06\right)$. After 4 weeks of RCT, absolute DWIbased tumor volumes of pathologically complete responders were significantly lower than for partial responders $\left(2.9 \mathrm{~cm}^{3}\right.$ vs. $\left.7.9 \mathrm{~cm}^{3}, p=0.03\right)$.

\section{ADC analyses}

When comparing patients with $\mathrm{pCR}$ and $\mathrm{pPR}$, the mean tumor ADC values at baseline as well as during the course of RCT widely overlapped; showing no statistically significant group difference for any ADC dynamics measures. The baseline average ADC values were comparable in both response groups (pCR: $1.04 \times 10^{-3} \mathrm{~mm}^{2} /$ $\mathrm{s}$, range $0.92-1.25 \times 10^{-3} \mathrm{~mm}^{2} / \mathrm{s}$; pPR: $1.04 \times 10^{-3} \mathrm{~mm}^{2} /$ $\mathrm{s}$, range $0.96-1.10 \times 10^{-3} \mathrm{~mm}^{2} / \mathrm{s} ; p=0.30$ ); during $\mathrm{RCT}$, the average ADC values at weeks 2 and 4 after initiation of RCT were $1.31 \times 10^{-3} \mathrm{~mm}^{2} / \mathrm{s}$ (range $1.22-1.37 \times 10^{-3}$ $\mathrm{mm}^{2} / \mathrm{s}$ ) and $1.43 \times 10^{-3} \mathrm{~mm}^{2} / \mathrm{s}$ (range $1.38-1.50 \times 10^{-3}$ $\mathrm{mm}^{2} / \mathrm{s}$ ) in the $\mathrm{pCR}$ group and $1.33 \times 10^{-3} \mathrm{~mm}^{2} / \mathrm{s}$ (range $1.21-1.46 \times 10^{-3} \mathrm{~mm}^{2} / \mathrm{s}$ ) and $1.44 \times 10^{-3} \mathrm{~mm}^{2} / \mathrm{s}$ (range $\left.1.27-1.67 \times 10^{-3} \mathrm{~mm}^{2} / \mathrm{s}\right)$ in the pPR group $(p=0.60$ and $p=0.70$ ). The detailed course of ADC values during neoadjuvant RCT is summarized in Table 4 .

\section{Discussion}

For the first time this exploratory study prospectively evaluated the predictive value of fractional MRI examinations for the early assessment of regression patterns in rectal cancer patients during neoadjuvant RCT.

In our analysis, T2-based tumor volume at baseline as well as at weeks 2 and 4 (fraction 11 and 22) of neoadjuvant RCT significantly correlated with the patients' $\mathrm{pCR}$ rates as assessed post surgery. In this context, a consistent tumor shrinkage relative to the initial tumor volume was observed during RCT, whereby the most considerable changes manifested in the first 2 weeks of treatment. Until the end of RCT, the average T2-tumor volume decreased by $87 \%$ in the pCR group and by $58 \%$ in $\mathrm{pPR}$ patients. When daily rectal tumor volume changes were compared to the volume measured during the previous treatment fraction, average tumor shrinkage rates steadily increased in the pCR group while they decreased in the pPR group. Our data support the hypothesis that a pCR may be predicted early during neoadjuvant RCT based on MRI patterns as tumor volume thresholds of below $25 \mathrm{ml}$ after 2 weeks and $10 \mathrm{ml}$ after 4 weeks correlated with the achievement of a postRT pCR. However, the proposed threshold values should be considered with caution due to the small number of patients in our analysis, which need to be verified in larger future studies.

Several analyses based on MRI suggested tumor volume reductions of $70-75 \%$ from baseline until re-staging 
Table 3 Comparison of DWI-based tumor volumetry between response groups

\begin{tabular}{|c|c|c|c|c|c|c|c|c|c|c|}
\hline & \multicolumn{10}{|c|}{ DWI-based tumor volumetry (ml) } \\
\hline & \multicolumn{5}{|l|}{$\overline{\mathrm{pCR}}$} & \multicolumn{5}{|l|}{$\mathrm{pPR}$} \\
\hline & Mean & Min & Max & Mean rel. to BL (\%) & $\bar{n}$ & Mean & Min & Max & Mean rel. to $\mathrm{BL}(\%)$ & 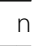 \\
\hline Fx 1 & 7.8 & 5.4 & 11.6 & 100 & 3 & 23.4 & 5.9 & 38.3 & 100 & 2 \\
\hline Fx 2 & 5.5 & 5.3 & 5.7 & 91.2 & 2 & 17.3 & 6.4 & 31.5 & 78.4 & 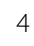 \\
\hline Fx 3 & 7.6 & 5.6 & 10.4 & 107.7 & 4 & 32.6 & 26.0 & 39.2 & 91.0 & 2 \\
\hline Fx 4 & 4.2 & 3.0 & 5.2 & 46.5 & 3 & 13.2 & 6.1 & 26.6 & 74.1 & 3 \\
\hline Fx 5 & 4.7 & 2.7 & 6.2 & 51.8 & 3 & 11.3 & 3.5 & 25.2 & 52.2 & 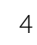 \\
\hline Fx 6 & 4.4 & 2.9 & 5.8 & 58.7 & 3 & 13.8 & 9.4 & 18.2 & 38.2 & 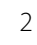 \\
\hline Fx 7 & 5.2 & 3.2 & 6.7 & 75.5 & 3 & 11.5 & 5.7 & 18.4 & 38.0 & 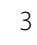 \\
\hline Fx 8 & 4.7 & 2.3 & 7.1 & 65.8 & 4 & 18.5 & 16.1 & 20.8 & 51.9 & 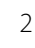 \\
\hline Fx 9 & 4.0 & 1.8 & 6.7 & 60.3 & 4 & 7.6 & 7.6 & 7.6 & 128.5 & 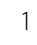 \\
\hline Fx 10 & 4.2 & 2.1 & 6.7 & 66.2 & 4 & 14.4 & 5.5 & 30.0 & 72.8 & 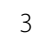 \\
\hline Fx 11 & 4.6 & 2.5 & 7.2 & 70.5 & 4 & 11.9 & 4.5 & 21.3 & 54.9 & 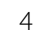 \\
\hline Fx 12 & 3.8 & 1.8 & 5.6 & 52.3 & 3 & 6.7 & 6.6 & 6.8 & 75.9 & 2 \\
\hline Fx 13 & 3.9 & 2.6 & 6.5 & 76.6 & 3 & NA & - & - & NA & 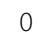 \\
\hline Fx 14 & 4.5 & 1.1 & 7.3 & 79.1 & 4 & 13.7 & 4.4 & 19.0 & 78.0 & 3 \\
\hline Fx 15 & 3.6 & 1.7 & 5.7 & 58.1 & 4 & 14.4 & 4.3 & 24.6 & 68.1 & 2 \\
\hline Fx 16 & 3.5 & 0.9 & 6.7 & 53.3 & 4 & 9.7 & 3.7 & 14.4 & 46.7 & 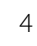 \\
\hline Fx 17 & 3.7 & 1.2 & 7.7 & 57.2 & 3 & 7.8 & 2.4 & 13.3 & 40.5 & 3 \\
\hline Fx 18 & 2.5 & 1.2 & 3.4 & 31.7 & 3 & 9.3 & 3.0 & 15.6 & 45.3 & 2 \\
\hline Fx 19 & 3.4 & 1.5 & 7.2 & 57.6 & 4 & 8.0 & 3.3 & 12.7 & 44.4 & 2 \\
\hline Fx 20 & 3.7 & 1.2 & 6.0 & 65.4 & 4 & 5.5 & 5.4 & 5.5 & 62.3 & 2 \\
\hline Fx 21 & 3.4 & 1.0 & 6.7 & 54.9 & 4 & 5.6 & 3.5 & 7.3 & 39.8 & 3 \\
\hline Fx 22 & 2.9 & 1.1 & 5.3 & 47.0 & 4 & 7.9 & 5.1 & 10.0 & 46.4 & 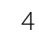 \\
\hline Fx 23 & 3.3 & 2.2 & 4.6 & 66.3 & 3 & 8.1 & 4.1 & 12.1 & 50.1 & 2 \\
\hline Fx 24 & 3.5 & 1.6 & 6.7 & 60.6 & 3 & 4.5 & 3.3 & 5.7 & 44.6 & 2 \\
\hline Fx 25 & 2.8 & 1.4 & 5.1 & 43.4 & 3 & 6.8 & 3.8 & 9.8 & 43.2 & 3 \\
\hline Fx 26 & 1.6 & 1.1 & 2.4 & 26.8 & 3 & 9.1 & 3.5 & 12.1 & 42.1 & 3 \\
\hline Fx 27 & 2.7 & 0.7 & 6.6 & 52.1 & 4 & 10.1 & 6.1 & 14.0 & 36.6 & 2 \\
\hline Fx 28 & 2.4 & 0.7 & 5.8 & 44.4 & 4 & 8.4 & 4.2 & 16.2 & 42.5 & 3 \\
\hline
\end{tabular}

Abbreviations: $m /$ milliliters, $p C R$ pathological complete response, $p P R$ pathological partial response, rel. relative, $B L$ baseline, $M i n$ minimum, $M a x$ maximum, $F x$ fraction, NA not analyzable, i.e. no values existent

prior to surgery to be associated with a pCR [24-26], and a recent observational study with weekly MRI reported similar tumor volume changes as compared to those we found in this analysis, supporting the predictive value of tumor volume regression during RCT [15].

Further published data investigating the predictive power of ADC changes in the context of neoadjuvant treatment showed conflicting results [27-30]. Intven et al. proposed an initial ADC value of $0.97 \times 10^{-3} \mathrm{~mm}^{2} /$ $\mathrm{s}$ and an ADC difference of $41 \%$ between pre-RCT and pre-surgery MRI scans as a cut-off for differentiation between good and moderate/poor responders [17]. In contrast, ADC values could not predict pathological treatment response in our analysis as ADC values widely overlapped between complete and non-complete responders during RCT. In this context, the small number of patients in our prospective study has to be considered concerning the interpretation of results as single outliers may already distort the statistical analysis. Nevertheless, our data do not support the suggested predictive value of delta ADC measurements during RCT.

Several studies analyzed a predictive potential of DWI volumetry for the identification of rectal cancer patients with a pCR [30]. One available study proposed that DWI volumetry after RCT offered the best results for the prediction of pCR with a sensitivity of $70 \%$ and a specificity of $98 \%$ [31]. To the best of our knowledge, no data concerning daily DWI volumetry during RCT have been 
Table 4 Comparison of ADC-values between response groups

\begin{tabular}{|c|c|c|c|c|c|c|c|c|c|c|}
\hline & \multicolumn{10}{|c|}{ ADC values $\left(10^{-3} \mathrm{~mm}^{2} / \mathrm{s}\right)$} \\
\hline & \multicolumn{5}{|l|}{$\mathrm{pCR}$} & \multicolumn{5}{|l|}{$\mathrm{pPR}$} \\
\hline & Mean & Min & Max & Mean rel. to BL (\%) & $n$ & Mean & Min & Max & Mean rel. to BL (\%) & $\mathrm{n}$ \\
\hline Fx 1 & 1.04 & 0.92 & 1.25 & 100 & 3 & 1.04 & 0.96 & 1.10 & 100 & 4 \\
\hline Fx 2 & 1.10 & 0.89 & 1.32 & 105.1 & 2 & 1.02 & 0.95 & 1.08 & 97.6 & 4 \\
\hline $\mathrm{F} \times 3$ & 0.98 & 0.93 & 1.12 & 96.2 & 4 & 1.04 & 1.02 & 1.05 & 98.5 & 2 \\
\hline Fx 4 & 0.99 & 0.90 & 1.06 & 104.5 & 3 & 1.09 & 1.00 & 1.17 & 105.9 & 3 \\
\hline Fx 5 & 1.13 & 0.98 & 1.34 & 127.9 & 3 & 1.07 & 1.01 & 1.19 & 102.9 & 4 \\
\hline Fx 6 & 1.17 & 1.05 & 1.24 & 116.0 & 3 & 1.12 & 1.11 & 1.12 & 106.0 & 2 \\
\hline Fx 7 & 1.18 & 1.05 & 1.30 & 106.5 & 3 & 1.16 & 1.09 & 1.20 & 108.3 & 3 \\
\hline Fx 8 & 1.19 & 1.09 & 1.29 & 116.4 & 4 & 1.20 & 1.14 & 1.27 & 114.0 & 2 \\
\hline Fx 9 & 1.24 & 1.14 & 1.39 & 124.6 & 4 & 1.30 & 1.30 & 1.30 & 134.9 & 1 \\
\hline Fx 10 & 1.21 & 1.13 & 1.29 & 119.1 & 4 & 1.29 & 1.20 & 1.35 & 126.0 & 3 \\
\hline Fx 11 & 1.31 & 1.22 & 1.37 & 127.7 & 4 & 1.33 & 1.21 & 1.46 & 127.8 & 4 \\
\hline Fx 12 & 1.31 & 1.24 & 1.39 & 125.6 & 3 & 1.26 & 1.23 & 1.30 & 123.4 & 2 \\
\hline Fx 13 & 1.24 & 1.13 & 1.38 & 118.8 & 3 & NA & - & - & NA & 0 \\
\hline Fx 14 & 1.36 & 1.26 & 1.44 & 133.6 & 4 & 1.30 & 1.24 & 1.39 & 127.5 & 3 \\
\hline Fx 15 & 1.35 & 1.27 & 1.43 & 131.4 & 4 & 1.43 & 1.37 & 1.48 & 143.9 & 2 \\
\hline Fx 16 & 1.40 & 1.29 & 1.50 & 137.3 & 4 & 1.34 & 1.23 & 1.52 & 128.7 & 4 \\
\hline Fx 17 & 1.38 & 1.26 & 1.55 & 135.5 & 3 & 1.33 & 1.23 & 1.46 & 129.9 & 3 \\
\hline Fx 18 & 1.45 & 1.30 & 1.55 & 151.9 & 3 & 1.34 & 1.24 & 1.44 & 135.1 & 2 \\
\hline Fx 19 & 1.38 & 1.32 & 1.43 & 133.1 & 4 & 1.36 & 1.28 & 1.43 & 137.0 & 2 \\
\hline Fx 20 & 1.33 & 0.98 & 1.50 & 123.2 & 4 & 1.35 & 1.21 & 1.48 & 132.3 & 2 \\
\hline Fx 21 & 1.52 & 1.38 & 1.69 & 150.0 & 4 & 1.31 & 1.25 & 1.44 & 128.6 & 3 \\
\hline Fx 22 & 1.43 & 1.36 & 1.50 & 138.0 & 4 & 1.44 & 1.27 & 1.67 & 138.2 & 4 \\
\hline Fx 23 & 1.43 & 1.39 & 1.49 & 129.4 & 3 & 1.40 & 1.39 & 1.40 & 140.8 & 2 \\
\hline Fx 24 & 1.47 & 1.35 & 1.55 & 137.8 & 3 & 1.37 & 1.26 & 1.48 & 134.5 & 2 \\
\hline Fx 25 & 1.44 & 1.38 & 1.50 & 140.5 & 3 & 1.36 & 1.26 & 1.51 & 133.6 & 3 \\
\hline Fx 26 & 1.41 & 1.36 & 1.49 & 145.3 & 3 & 1.53 & 1.43 & 1.65 & 150.2 & 3 \\
\hline Fx 27 & 1.51 & 1.46 & 1.54 & 147.1 & 4 & 1.35 & 1.27 & 1.43 & 127.3 & 2 \\
\hline Fx 28 & 1.50 & 1.38 & 1.60 & 143.7 & 4 & 1.53 & 1.45 & 1.63 & 150.1 & 3 \\
\hline
\end{tabular}

Abbreviations: $p C R$ pathological complete response, $p P R$ pathological partial response, rel. relative, $B L$ baseline, Min minimum, Max maximum, Fx fraction, NA not analyzable, i.e. no values existent

reported. In our analysis, significant differences of DWIbased tumor volumes between patients with $\mathrm{pCR}$ and pPR were observed at week 4 during neoadjuvant RCT. In contrast, neither the baseline DWI volumetry nor the relative volume decreases showed a significant difference between both response groups in our dataset. Our data suggest that pathological response can be predicted by DWI volumetry during neoadjuvant treatment, although our findings have to be corroborated in larger patient cohorts. In addition, this analysis revealed significant differences in T2 and DWI volumetry, which may be explained on the one hand by the difference between tumor volumetry based on morphological MRI sequences as compared to functional DWI and on the other hand by the semi-automatic segmentation approach in case of DWI.

The detailed description of daily volumetric changes in this study aims to serve as a template to design further trials to confirm the role of MRI as a means for early response assessment in order to devise response-specific adaptive treatment strategies. Tumor volume decreases during RCT offer the option to adapt treatment volumes for a better sparing of the surrounding tissues-at-risk with a potential impact on acute and late radiogenic toxicities as well as perioperative morbidity [15]. There is an increasing interest in tailoring neoadjuvant treatments more closely to the extent of tumor regression during RCT based on MRI examinations [32]. Therefore, 
MR parameters have to be defined for reliable differentiation between good and moderate or poor responders during neoadjuvant treatment $[15,16]$. Particularly poor and moderate responders may benefit from dose escalation strategies in order to increase local control and survival rates. However, the implementation of adaptive approaches in the daily routine is difficult for practical reasons. On the other hand, adaptive radiotherapy (ART) including boost strategies might result in higher clinical complete response rates (cCR), which has been suggested to influence progression-free-survival [33]. In recent years, RCT with consecutive $\mathrm{CCR}$ or $\mathrm{pCR}$ may allow wait-and-watch approaches or local tumor excision strategies, thereby sparing patients mutilating surgeries and improving treatment-related toxicities and quality of life $[9,34,35]$. While standard RCT regimes result in CCR rates of about $17-28 \%$, considerably higher cCR rates up to $70 \%$ seem to be achievable by higherdose RCT approaches [9, 36-39].

This study mainly serves as an accurate template for determining appropriate measurement times for DWI and volumetric monitoring during RCT that may be applicable to novel MRI-guided radiotherapy approaches. This is of special interest for treatment concepts using hybrid MRI-linear accelerators, enabling integrated daily fractional MRI [40-42]. However, it is important to note the different capabilities of diagnostic MRI (as performed in this prospective study) and imaging provided by hybrid devices, especially with regard to DWI requiring b0 homogeneity [43].

Despite the complex and comprehensive MR imaging protocols used here, the small number of patients limits our analysis, and corroboration of our findings in a larger patient cohort is warranted. Furthermore, the sample is not fully representative of the general population, as the pCR rate in our dataset amounted to $50 \%$; nevertheless, as the focus of our analysis was to analyze and report the longitudinal course of MR regression patterns during neoadjuvant treatment in detail, we feel that this deviation of the pCR rates from previous cohorts should not invalidate our data $[44,45]$. This analysis does not include inter-observer evaluation, which could be a potentially important factor, since reported interfractional volumetric differences during neoadjuvant treatment could be within the range of expected differences between independent observers. To date, no other data are available reporting daily anatomical and functional MR data for rectal cancer patients undergoing neoadjuvant radiochemotherapy. Therefore, this study provides a unique comprehensive dataset, which can help to gain valuable new insights into detailed MR regression patterns during neoadjuvant treatment of rectal cancers and the trends observed from this data may serve as a useful template for future MR-guided radiotherapy studies.

\section{Conclusion}

This prospective study supports the hypothesis that MRI may be able to predict $\mathrm{pCR}$ of rectal cancers early during neoadjuvant RCT. Our data therefore provide a useful template to tailor future MR-guided adaptive treatment concepts.

\section{Abbreviations \\ MRI: Magnetic resonance imaging; MR: Magnetic resonance; RCT: Radiochemotherapy; RT: Radiotherapy; ART: Adaptive radiotherapy; DWI: Diffusion-weighted-imaging; ADC: Apparent diffusion coefficient; pCR: Pathological complete response; pPR: Pathological partial response; CCR: Clinical complete response; GTV : Gross tumor volume; CTV: Clinical target volume; PTV: Planning target volume; MV: Megavolt; KV: Kilovolt; T : Tesla; TR: Time of repetition; TE : Time of echo; $\mathrm{mm}$ : Millimeter; cm: Centimeter; ms: Milliseconds; s: Seconds; FOV: Field of view; ROI: Region of interest; MITK: Medical Imaging Interaction Toolkit}

\section{Acknowledgements}

We thank Diacor Inc. for supplying the Zephyr system, which was used in this study. The authors acknowledge the contribution of Dr. Peter Haering, Clemens Lang, Mona Splinter and Dr. Asja Pfaffenberger, who substantially contributed to this study by the acquisition and processing of patient data.

\section{Authors' contributions}

TB, CD, FS, JD and NHN developed and planned this prospective analysis. DW is responsible for statistical considerations/basis of the analysis. TB, CD AM, FK, SB, HPS, PEH, FS, PB, JD and NHN participated in data collection and interpretation of the results. TB and CD wrote the manuscript. All authors read and approved the final manuscript.

\section{Funding}

This study project was funded by the Federal Ministry of Education and Research of Germany (BMBF), Grant No. 01/B13001 (SPARTA).

\section{Availability of data and materials}

The datasets used and/or analysed during the current study are available from the corresponding author on reasonable request.

\section{Ethics approval and consent to participate}

The Heidelberg Ethics Committee approved this study prior to patient recruitment (S-144/2013). All patients treated in this prospective study signed a written informed consent, which included the agreement for scientific use of their data.

\section{Consent for publication}

Not applicable.

\section{Competing interests}

S.B. receives lecture/travel-fee from Siemens due to (pending) patents in DWI. The Universitätsklinikum Erlangen as the employer of S.B. receives financial funding by Siemens Healthineers which is partially used as well for his job position. The rest of the authors declare that they have no competing interests.

\footnotetext{
Author details

${ }^{1}$ Clinical Cooperation Unit Radiation Oncology, German Cancer Research Center (DKFZ), Im Neuenheimer Feld 280, 69120 Heidelberg, Germany. ${ }^{2}$ Department of Radiation Oncology, University Medical Center Mainz, Langenbeckstrasse 1, 55131 Mainz, Germany. ${ }^{3}$ Division of Radiology, German Cancer Research Center (DKFZ), Im Neuenheimer Feld 280, 69120 Heidelberg, Germany. ${ }^{4}$ Department of Radiation Oncology, University Hospital Mannheim, Medical Faculty Mannheim, Heidelberg University, Theodor Kutzer-Ufer 1-3, 68167 Mannheim, Germany. ${ }^{5}$ Institute of Medical Biostatistics, Epidemiology and Informatics (IMBEI), University Medical Center Mainz, Obere Zahlbacher Strasse 69, 55131 Mainz, Germany. ${ }^{6}$ Division of Medical Imaging and Radiology - Cancer Prevention, German Cancer Research Center (DKFZ), Im Neuenheimer Feld 280, 69120 Heidelberg, Germany. ${ }^{7}$ Institute of Radiology, Friedrich-Alexander-University Erlangen-Nürnberg, Maximiliansplatz 2, 91054 Erlangen, Germany.
} 
${ }^{8}$ Department of Radiation Oncology, University Hospital of Heidelberg, Im Neuenheimer Feld 400, 69120 Heidelberg, Germany. ${ }^{9}$ Radiation Oncology, Kempten Clinic, Robert-Weixler-Strasse 50, 87439 Kempten, Germany.

${ }^{10}$ dia.log, Altoetting Center for Radiology, Vinzenz-von-Paul-Strasse 10, 84503 Altoetting, Germany. ${ }^{11}$ Department of Radiation Oncology, University of Freiburg Medical Center, Robert-Koch-Strasse 3, 79106 Freiburg, Germany.

Received: 13 January 2020 Accepted: 3 July 2020

Published online: 11 July 2020

\section{References}

1. Haggar FA, Boushey RP. Colorectal cancer epidemiology: incidence, mortality, survival, and risk factors. Clin Colon Rectal Surg. 2009;22(4): 191-7.

2. Brenner $H$, Stock $C$, Hoffmeister M. Effect of screening sigmoidoscopy and screening colonoscopy on colorectal cancer incidence and mortality: systematic review and meta-analysis of randomised controlled trials and observational studies. BMJ. 2014;348:g2467.

3. Levin B, et al. Screening and surveillance for the early detection of colorectal cancer and adenomatous polyps, 2008: a joint guideline from the American Cancer Society, the US multi-society task force on colorectal cancer, and the American College of Radiology. CA Cancer J Clin. 2008; 58(3):130-60.

4. van Gijn W, et al. Preoperative radiotherapy combined with total mesorectal excision for resectable rectal cancer: 12-year follow-up of the multicentre, randomised controlled TME trial. Lancet Oncol. 2011;12(6):575-82.

5. Camma C, et al. Preoperative radiotherapy for resectable rectal cancer: a meta-analysis. JAMA. 2000;284(8):1008-15.

6. Gerard JP, et al. Preoperative radiotherapy with or without concurrent fluorouracil and leucovorin in T3-4 rectal cancers: results of FFCD 9203. Clin Oncol. 2006;24(28):4620-5.

7. Bosset JF, et al. Chemotherapy with preoperative radiotherapy in rectal cancer. N Engl J Med. 2006;355(11):1114-23.

8. Janjan NA, et al. Prospective trial of preoperative concomitant boost radiotherapy with continuous infusion 5-fluorouracil for locally advanced rectal cancer. Int J Radiat Oncol Biol Phys. 2000;47(3):713-8.

9. Habr-Gama A, et al. Operative versus nonoperative treatment for stage 0 distal rectal cancer following chemoradiation therapy: long-term results. Ann Surg. 2004;240(4):711-7 discussion 717-8.

10. Maas $M$, et al. Wait-and-see policy for clinical complete responders after chemoradiation for rectal cancer. J Clin Oncol. 2011;29(35):4633-40.

11. Maas $\mathrm{M}$, et al. Long-term outcome in patients with a pathological complete response after chemoradiation for rectal cancer: a pooled analysis of individual patient data. Lancet Oncol. 2010;11(9):835-44.

12. Patel UB, et al. Magnetic resonance imaging-detected tumor response for locally advanced rectal cancer predicts survival outcomes: MERCURY experience. J Clin Oncol. 2011;29(28):3753-60.

13. Chiloiro G, et al. MR-guided radiotherapy in rectal cancer: first clinical experience of an innovative technology. Clin Transl Radiat Oncol. 2019; 18:80-6.

14. Gani C, Boldrini L, Valentini V. Online MR guided radiotherapy for rectal cancer. New opportunities. Clin Transl Radiat Oncol. 2019;18:66-7.

15. Van den Begin $R$, et al. Tumor volume regression during preoperative chemoradiotherapy for rectal cancer: a prospective observational study with weekly MRI. Acta Oncol. 2018;57(6):723-7.

16. Bakke KM, et al. Diffusion-weighted magnetic resonance imaging of rectal cancer: tumour volume and perfusion fraction predict chemoradiotherapy response and survival. Acta Oncol. 2017;56(6):813-8.

17. Intven $M$, Reerink $O$, Philippens ME. Diffusion-weighted MRI in locally advanced rectal cancer : pathological response prediction after neoadjuvant radiochemotherapy. Strahlenther Onkol. 2013;189(2):117-22.

18. Boldrini $L$, et al. Delta radiomics for rectal cancer response prediction with hybrid $0.35 \mathrm{~T}$ magnetic resonance-guided radiotherapy (MRgRT): a hypothesis-generating study for an innovative personalized medicine approach. Radiol Med. 2019;124(2):145-53.

19. Bostel T, et al. Prospective feasibility analysis of a novel off-line approach for MR-guided radiotherapy. Strahlenther Onkol. 2018;194(5):425-34.

20. Bostel T, et al. MR-guidance--a clinical study to evaluate a shuttlebased MR-linac connection to provide MR-guided radiotherapy. Radiat Oncol. 2014;9:12.
21. Dworak O, Keilholz L, Hoffmann A. Pathological features of rectal cancer after preoperative radiochemotherapy. Int J Color Dis. 1997;12(1):19-23.

22. Valentini $V$, et al. International consensus guidelines on clinical target volume delineation in rectal cancer. Radiother Oncol. 2016;120(2):195-201.

23. Nolden $\mathrm{M}$, et al. The medical imaging interaction toolkit: challenges and advances : 10 years of open-source development. Int J Comput Assist Radiol Surg. 2013;8(4):607-20.

24. Kang JH, et al. Tumor volume changes assessed by three-dimensional magnetic resonance volumetry in rectal cancer patients after preoperative chemoradiation: the impact of the volume reduction ratio on the prediction of pathologic complete response. Int J Radiat Oncol Biol Phys. 2010;76(4): 1018-25.

25. Barbaro B, et al. Locally advanced rectal cancer: MR imaging in prediction of response after preoperative chemotherapy and radiation therapy. Radiology. 2009;250(3):730-9.

26. Dresen $\mathrm{RC}$, et al. Locally advanced rectal cancer: MR imaging for restaging after neoadjuvant radiation therapy with concomitant chemotherapy. Part I. are we able to predict tumor confined to the rectal wall? Radiology. 2009; 252(1):71-80

27. Jacobs $L$, et al. Diffusion-weighted MRI for early prediction of treatment response on preoperative Chemoradiotherapy for patients with locally advanced rectal cancer: a feasibility study. Ann Surg. 2016;263(3):522-8.

28. Barbaro B, et al. Diffusion-weighted magnetic resonance imaging in monitoring rectal cancer response to neoadjuvant chemoradiotherapy. Int J Radiat Oncol Biol Phys. 2012;83(2):594-9.

29. Lambrecht $M$, et al. Value of diffusion-weighted magnetic resonance imaging for prediction and early assessment of response to neoadjuvant radiochemotherapy in rectal cancer: preliminary results. Int J Radiat Oncol Biol Phys. 2012;82(2):863-70.

30. Curvo-Semedo L, et al. Rectal cancer: assessment of complete response to preoperative combined radiation therapy with chemotherapy--conventional MR volumetry versus diffusion-weighted MR imaging. Radiology. 2011; 260(3):734-43.

31. Lambregts DM, et al. MRI and diffusion-weighted MRI Volumetry for identification of complete tumor responders after preoperative Chemoradiotherapy in patients with rectal cancer: a bi-institutional validation study. Ann Surg. 2015;262(6):1034-9.

32. Van Wickle JD, et al. Adaptive radiation dose escalation in rectal adenocarcinoma: a review. J Gastrointest Oncol. 2017;8(5):902-14.

33. Lim L, et al. Long-term outcomes of patients with localized rectal cancer treated with chemoradiation or radiotherapy alone because of medical inoperability or patient refusal. Dis Colon Rectum. 2007:50(12):2032-9.

34. Habr-Gama A. Assessment and management of the complete clinical response of rectal cancer to chemoradiotherapy. Color Dis. 2006;8(Suppl 3):21-4.

35. Renehan $A G$, et al. Watch-and-wait approach versus surgical resection after chemoradiotherapy for patients with rectal cancer (the OnCoRe project): a propensity-score matched cohort analysis. Lancet Oncol. 2016;17(2):174-83.

36. Appelt AL, et al. High-dose chemoradiotherapy and watchful waiting for distal rectal cancer: a prospective observational study. Lancet Oncol. 2015; 16(8):919-27.

37. Habr-Gama A, et al. Long-term results of preoperative chemoradiation for distal rectal cancer correlation between final stage and survival. J Gastrointest Surg. 2005;9(1):90-9 discussion 99-101.

38. Habr-Gama A, et al. Patterns of failure and survival for nonoperative treatment of stage c0 distal rectal cancer following neoadjuvant chemoradiation therapy. J Gastrointest Surg. 2006;10(10):1319-28 discussion 1328-9.

39. Hughes R, Harrison M, Glynne-Jones R. Could a wait and see policy be justified in T3/4 rectal cancers after chemo-radiotherapy? Acta Oncol. 2010; 49(3):378-81.

40. Mutic S, Dempsey JF. The ViewRay system: magnetic resonance-guided and controlled radiotherapy. Semin Radiat Oncol. 2014;24(3):196-9.

41. Raaymakers BW, et al. Integrating a $1.5 \mathrm{~T}$ MRI scanner with a $6 \mathrm{MV}$ accelerator: proof of concept. Phys Med Biol. 2009;54(12):N229-37.

42. Klüter S, Katayama S, Spindeldreier CK, et al. First prospective clinical evaluation of feasibility and patient acceptance of magnetic resonanceguided radiotherapy in Germany. Strahlenther Onkol. 2020. https://doi.org/ 10.1007/s00066-020-01578-Z.

43. Yang $Y$, et al. Longitudinal diffusion MRI for treatment response assessment: preliminary experience using an MRI-guided tri-cobalt 60 radiotherapy system. Med Phys. 2016;43(3):1369-73. 
44. Sauer $\mathrm{R}$, et al. Preoperative versus postoperative chemoradiotherapy for rectal cancer. N Engl J Med. 2004;351(17):1731-40.

45. Cercek A, et al. Adoption of Total neoadjuvant therapy for locally advanced rectal cancer. JAMA Oncol. 2018;4(6):e180071.

\section{Publisher's Note}

Springer Nature remains neutral with regard to jurisdictional claims in published maps and institutional affiliations.

Ready to submit your research? Choose BMC and benefit from:

- fast, convenient online submission

- thorough peer review by experienced researchers in your field

- rapid publication on acceptance

- support for research data, including large and complex data types

- gold Open Access which fosters wider collaboration and increased citations

- maximum visibility for your research: over $100 \mathrm{M}$ website views per year

At BMC, research is always in progress.

Learn more biomedcentral.com/submissions 